\title{
INVESTIGACION
}

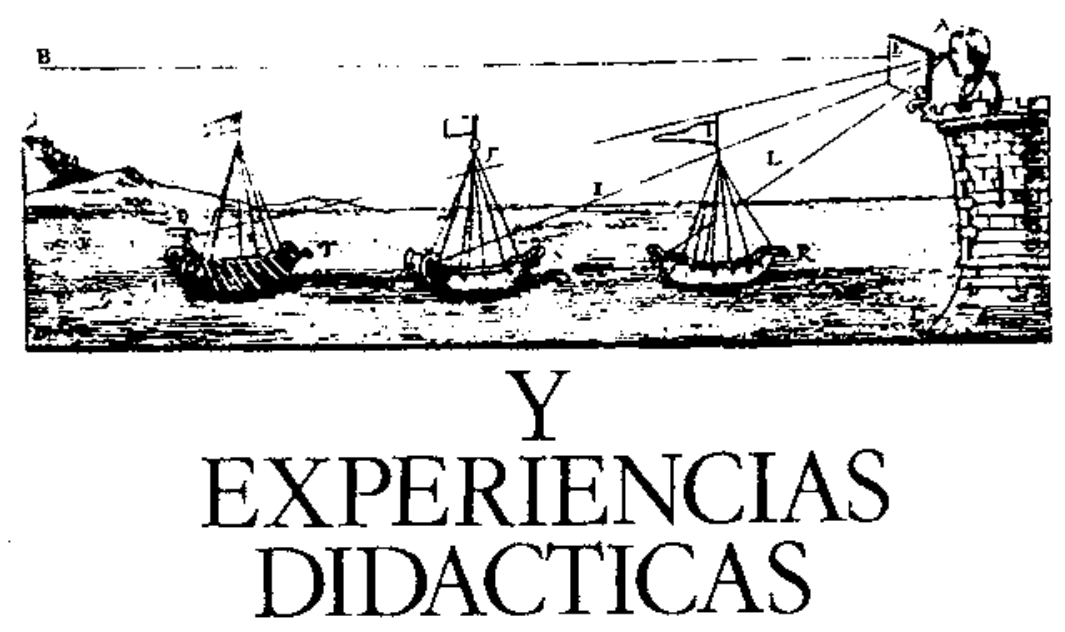

\section{IDEAS SOBRE LOS GASES EN ALUMNOS DE 10 A 15 AÑOS}

FURIO MAS, C. y HERNANDEZ PEREZ, I.

l.C.E. Universidad de Valencia.

\section{SUMMARY}

This paper shows some ideas the students have on specific perceptive aspects of gases, such as their materiality or the volume they take, together with the analysis of their answers to their internal structure.

The results found are similar to those obtained from other coniext. It is confirmed that students maintain an uristotelic vision of the substantiality of gases, though some features of the particulate theory (accepted at present as for (heir internai structure) equally appear.

\section{INTRODUCCION}

Cualquier metodologia de enseñanza de las ciencias que pretenda conseguir un aprendizaje significativo debe necesariamente partir de las ideas, concepciones o preconceptos que el alumno posee en el momento de abordar los problemas referentes a una temática concreta. En este sentido Ausube] (1978) al tratar de las consecuencias generales para la educación, de las etapas de desarrollo mental en el ninto, indica que:

«Conocer el plan que sigue el desarrolio intelectual hace factible, por primera vez, la ubicacion cientifica de la materia de estudio, que contrasta con la ubicación arbitraria o tradicional..."

Ahora bien, aquellas ideas o concepciones que caracterizan la estructura cognoscitiva del alumno, no se presentan de forma inconexa o comparimentada, sino que constituyen un tejido o cuerpo de conocimientos de partida con los cuales interpretan el mundo exterior ( $\mathrm{Ca}$. rrascosa y Gil 1982) y al que podemos denominar pa. radigma conceptual discente. Asi pués, la labor básica del profesor principia por conocer este paradigma con- 
ceptual del alumno para entender sus posibles errores conceptuales y situar en este marco su propuesta de acción metodológica; y con mayor razón, si desea utilizar una metodología de descubrimiento guiado que sea coherente con la psicología educativa y con ta metología cientifica.

Puede suceder que el esquema conceptual del alumno sea compatible con los nuevos conocimientos cientifi$\cos$ que se deseen impartir o redescubrir en la clase, en este caso, aquellas ideas podrán ser explotadas y podrán actuar como principio organizador de futuras profundizaciones. En caso contrario, una actitud positiva del profesor consistiria en valorar aquellas ideas equivocadas y presentar el conflicto cognoscitivo mediante el oportuno debate o interposicion de actividades que demuestren al alumno su incoherencia conceptual (Hewson, 1981).

Cuando se inicia el estudio del comportamiento quj. mico de la materia en el Bachillerato, es de sumo inte. rés conocer las ideas que poseen los alumnos sobre los gases, con el fin de tener un punto de partida para la construcción del curriculum de Quimica y, sobre to. do, para el estudio de la teoria atómico-molecular de la materia. En consecuencia, en este trabajo se planteó la indagación sobre el grado de asunción de nuestra concepción actual de gas en dos vertientes. Una de ellas se referia a qué pensaban los alumnos sobre lo que era un gas y una segunda, perteneciente al mundo no perceptivo, destinada a extraer el pensamiento del alumnado sobre cómo es un gas, es decir, cuál es su estructura interna.

\section{EMISION DE HIPOTESIS}

El cuerpo teórico de la psicologia educativa que aceptamos previamente en nuestro trabajo, es el conjunto de hipótesis emitidas por prestigiosos epistemólogos e historiadores de la ciencia (Piaget, 1975) (Holton, 1973), según las cuales se reconoce la existencia de cierto paralelismo entre el desarrollo cognoscitivo en el alumno y el modo de producción de los conocimientos cientificos (Kuhn, 1970). Dentro de este contexto teórico, nuestras suposiciones parten de admitir que los alumnos de edades comprendidas entre 12 y 15 attos que corresponden al periodo de las operaciones formales en sentido piagetiano, aún teniendo asumido un atomismo inicial lo que supone haber asimilado jerárqujcamente la conservación de la sustancia, del peso y del volumen, como lo demuestran los experimentos realizados sobre todo en sólidos y liquidos, con niños ginebrinos (Piaget e Inhelder, 1971) no responden como atomistas cuando se plantean problemas similares con los gases. Más en concreto, nuestra hipótesis implica que, desde el punto de vista perceptivo, muchos alumnos tienen la idea de los gases como algo casi inmateriat, con poca sustancialidad, etc... al igual que sucedió en la comunidad científica en la denominada prehistoria próxima de la Qufmica como ciencia moderna (Taton, 1958) (Davy, 1976) y que, en consecuencia, los alumnos están más próximos a las ideas aristotélicoescolásticas respecto a los gases, cuanto más se aproximan en edad a la transición entre la etapa de las operaciones formales y la de las operaciones concretas (1) o 12 años).

En resumen y de forma análoga a lo que sucede con el razonamiento espontáneo de los ninos respecto a la mecánica, cuyo esquema conceptual tiene una componente aristotélico-escolástica en su forma de ver el mundo, como ha sido demostrado por diferentes autores de diversos países (Viennot 1979) (Helm 1980) (Carrascosa, Gil y González 1982), así en el campo de la quimica y concretamente en su concepcion de los gases como materia, opinamos que se puede extrapolar aquella vision al comportamiento químico de la materia. Sin embargo, esta idea casi-inmaterial de los gases no implica que no contengan aspectos del modelo cinético que corresponden a una visión científica actual de los gases.

\section{DISEÑO EXPERIMENTAL}

Con el fin de comprobar la hipotesis anterior se encuestó a diferentes grupos de edades comprendidas entre 11 y 15 ahos como se indica a continuación.

\subsection{Alumnos Encuestados}

Se inició el trabajo realizando algunas encuestas y entrevistas previas a alumnos de la edad más baja (10 u 11 años) con dos objetivos. El primero de ellos consistia en ver si se obtenian respuestas inteligibles para nosotros cuando se contesiaba directamente a la cuestión de la naturaleza material de un gas. Estas entrevistas nos orientaron hacia el segundo objetivo que consistia en la planificación de nuestro cuestionario de preguntas.

Antes de lanzarnos a una exploración extensiva del pensamiento escolar se pensó en un pretest inicial y para ello se eligieron como muestras de población escolar. que no pueden ser consideradas como representativas, a 10 grupos de alumnos desde $5^{\circ}$ de EGB hasta $2^{\circ}$ de BUP con edades promedio de 10 a 15 años, con el fin de utilizar como variable independiente la edad de aquellos.

La distribución y la cantidad de alumnos encuestados se mencionan a continuación:

\begin{tabular}{|lcccccc|}
\hline Cursu & $5^{\prime \prime}$ EGB & $6^{\circ}$ EGB & $7^{\circ}$ EGB & $8^{\circ}$ EGB & $1^{\circ}$ BUP & $2^{\circ}$ BUP \\
Edad/Antos & $(10-11)$ & $(11-12)$ & $(12-13)$ & $(13-14)$ & $(14-15)$ & $(15-16)$ \\
Nümero & 36 & 34 & 65 & 60 & 73 & 70 \\
\hline
\end{tabular}




\subsection{Material suministrado}

Como interesaba contrastar, por una parte, si los alumnos tenian o no una idea sustancial del gas, y por otra, analizar el modelo de gas asimilado, se pasó un conjunto de seis preguntas (tres destinadas a cómo percibian el gas y otras tres destinadas a su concepción respecto del modelo), todas ellas cerradas, de opción múltiple, incluida la respuesta "no lo sé» para evitar la aleatoriedad en las contestaciones. Las preguntas se encontrarán en el siguiente apartado.

Los objetivos especificos perseguidos en cada una de las cuestiones eran los siguientes:

Cuestión 1.- Dilucidar si concebian los gases como sustancias que pesan o por el contrario, se aceptaba la idea consistente en suponer quc el gas no pesaba, con lo que se estaria, según nuestra opinión, más próximo a las ideas aristotélicas.

Cuestión 2.- Analizar si los alumnos, coherentemente con los planteamientos aristotélicos, suponen que el peso es una propiedad accidental que depende del estado fisico de la sustancia, o, por el contrario, interpretan correctamente la conservación del peso en una trans. formación de agua en vapor, en un recinto hermético.

Cuestión 3.- Descubrir si el alumno dispone claramente de la idea que, en una vaporización, el gas obtenido ocupa todo el volumen del recipiente que contenia al liquido inicial vaporizado.

Anàlogamente se confeccionaron tres preguntas que cubrian sendos objetivos específicos y relativos al modelo de gas y cuyas contestaciones nos permitirian analizar:

Cuestión 4.- ¿Estructura atomista - particulas- o continua para los gases?

Cuestión 5.- Admiten los alumnos la existencia de huecos en el modelo de gas, o por el contrario, su idea se acerca más a un modelo "compacto» de particulas.

Cuestión 6.- Finalmente se trató de analizar en qué grado se aceptaba el movimiento caótico de las partículas de un gas, según nuestro modelo cinético.

\subsection{Administración del cuestionario}

En el momento de pasarles este pretest se encontraba presente uno de los autores del trabajo, comprobando la inexistencia de dificultades en la contestación al mismo. Este test se pasó en horas de clase ordinaria y sin previo aviso con el fin de no condicionar las respuestas, no dando tiempo límite a las contestaciones para una mayor tranquilidad del alumnado. Por otra parte, conviene indicar que los alumnos de $2^{\circ}$ de BUP no habian iniciado el estudio de la Química, en cambio, los alumnos de $8^{\circ}$ de EGB habian ya tratado el estudio de los gases en clase, aunque hacia bastante tiempo. Asunto este último que habrá que tener en cuenta al analizar los resultados encontrados.

\section{PRESENTACION Y ANALISIS DE LOS RESULTADOS ENCONTRADOS}

A continuación se presentan las respuestas dadas por los alumnos encuestados a cada una de las preguntas, adjuntándose para una mejor visualización global un diagrama de barras en el que figuran el total de las contestaciones incorrectas (TOT.EQUI.) frente al curso de cada grupo. Junto a los porcentajes totales de contestaciones equivocadas se aporta la desviación estandard $s$ de la muestra según el tratamiento estadistico ordinario (Garret 1971), que viene dada por la expresión:

$$
S=\sqrt{\frac{P(100 \cdot P)}{N}}
$$

donde $p$ indica el porcentaje total de desaciertos y $N$, el número de alumnos encuestados.

En la mayor parte de los casos se ha procedido también el análisis de las respuestas incorrectas mayoritarias que proporcionarán una idea de los eriores más frecuentes del alumnado.

La tabla 1 recoge los resultados encontrados a la primera cuestión, mientras la fig. 1 corresponde al diagrama de barras de estos mismos resultados. De su observación se comprueba que la mayor parte de los niños de 10 y 11 años $(65 \%)$ consideran que los gases no pesan, disminuyendo esta tendencia cuando se llega a la etapa de transición entre las operaciones concretas y tas formales piagetianas. Sin embargo, apenas hay diferencias significativas a partir de $8^{\circ} \mathrm{EGB}$. Caben aqui varias interpretaciones, entre las cuales podemos citar: o bien estos niños de edades más bajas no atribuyen apenas materialidad a los gases si consideramos que el peso es, para ellos, una medida de la cantidad de sustancia (en estas edades se confunde el peso con la masa) o bien la carencia de peso es sinónimo de ligereza relativa, en el sentido de elevarse por encima del agua, ya que van a su lugar natural según la concepción aristotélica (Gil, 1981). Para nosotros ambas interpretaciones son la misma, pues según Aristóteles (1973) las definiciones de ligereza y pesadez implican las tendencias naturales del movimiento de los cuerpos hacia los dos "extremos" contrarios respectivamente. Asi pues, estas propiedades (ligereza y pesadez), siempre relativas, son potencialidades innatas en la materia de manera que «cuanto más ligero es un cuerpo más forma - más cualidad - y' menos materia tiene menos cantidad-", como puede leerse en el capitulo $4^{\circ}$ del libro IV de la Fisica de aquel autor.

Esta interpretación es coherente con los resultados encontrados a la pregunta $2^{\prime}$ (tabla II y fig. 2), donde los niños de 10 a 11 años siguen contestando aproximadamente en el mismo porcentaje $(57 \%)$ que en una transformación de liquido en gas, el primero debe pesar más que el segundo. Sin embargo, no sucede lo mismo con los alumnos de edades superiores a 12 años, 
que opinan en porcentajes elevados que el liquido pesará más que el gas. Lo que sí parece conluyente es que lo que ocurre con el peso en esta transformación (va. porización) en recipiente cerrado, no está claro para la mayorla del alumnado, contrariamente a los resul. tados encontrados por Piaget e Inhelder (1971) al entrevistar a nif̂os sobre la conservación del peso en el experimento de la disolución del azúcar en agua.

Mención a parte merece la interpretación de la respuesta dada por un número minoritario de alumnos - el $7 \%$ en el sentido de considerar mayor el peso del gas que el del líquido (respuesta $2 b$ ), suponemos que piensan en la adición material que supondría la calefacción del liquido, lo que correspondería a una concepción del calor como sustancia:

Respecto a la $3^{*}$ pregunta relativa a conocer si los alumnos admiten que el gas ocupa todo el volumen del recipiente que lo contiene (tabla III y fig. 3), no se encuentran diferencias significativas entre los alumnos de diferentes edades, si bien es de destacar que la mayor parte de los alumnos errados se inclinan por la conservación del volumen $\rightarrow$ como era de esperar $\rightarrow$, lo que unido a su concepción de que el gas no pesa (debido a que se eleva), como se ha indicado en los comentarios a las preguntas $1^{\text {" }}$ y $2 "$, les lleva a contestar la respuesta $3 b$. En conclusión, puede anotarse que cuatro de cada diez alumnos, por término medio, no tienen claro que un gas ocupa todo el volumen del recipiente que lo contiene.

Finalmente en la tabla IV se ofrecen los resultados giobales de estas tres cuestiones, habiendose tabulado el porcentaje de alumnos por curso que se han equivocado en todas o en alguna pregunta de las planteadas, asi como la desviación estandard encontrada en cada una de ellas.

En cuanto a las respuestas dadas por los alumnos a la $4^{\text {* }}$ pregunta (tabla $V$ y fig. 4), puede observarse que la mayor parte de los alumnos se inclinan por una estructura atomista del gas, no obstante, no debe des. preciarse que, por término medio, 3 de cada 10 alumnos conciben a los gases con una estructura continua. De este análisis se exceptuan los alumnos de $5^{\circ}$ de EGB donde puede darse en hipótesis una aleatoriedad en las contestaciones. Parecen observarse anomalias en $6^{\circ}$ y $8^{\circ}$ de EGB, ya que sus porcentajes de equivocados son los más bajos. Ello puede explicarse, pues constatamos que en estos cursos, los profesores nos indicaron que hablan tratado los gases en el currículum del curso. Esto prueba, una vez más, que a medida que transcurre el tiempo el alumnado vuelve a sus preconceptos anteriores, como se comprueba tanto en $7^{\circ}$ de EGB como en $1^{\circ}$ y $2^{\circ}$ de BUP que no habian incidido en estas materias.

Tampoco se observa en la $S^{a}$ pregunta (tabla Vl y fig. 5) grandes diferencias entre los alumnos de 12 y 15 aros (se han exceptuado las respuestas de $5^{\circ}$ EGB ya que se distribuyen casi aleatoriamente), siendo mayoritaria la opción por la existencia de huecos entre las partículas del gas, no obstante hay casi un $40 \%$ de alumnos que o bien no son atomistas, en el sentido de seguir pensando en un modelo continuo para los gases (cerca de un $25 \%$ ), o bien, lo son pero con la particularidad de admitir un modelo estático al igual que sugeria Dalton en sus escritos (Nash, 1970). En efecto, según Nash, en un articulo manuscrito de Dalion titulado "On heat» (sobre el calor), fechado en 23 de mayo de 1806, al explicar la difusión entre gases, atribuye a cada partícula (átomo + celdilla de calórico) un tamaño diferente y en consecuencia que en un mismo volumen de gas, fuera oxigeno o nitrógeno, no debia existir el mismo numero de particulas, ya que estas ocupaban todo el volumen sin posibilidad de existencia de huecos.

Los resultados encontrados sobre la aceptación o no del movimiento caótico de las partículas del gas (pregunta $6^{2}$ ), se ofrecen en la tabla VIl y la figura 6 . Al igual que sucedia en ia cuestión anterior, los alumnos se decantan claramente hacia el modelo caótico de las particulas del gas, independientemente de la edad de los mismos, pues aproximadamente siete de cada diez admiten este movimiento al azar (contestación $6 \mathrm{c}$ ).

Finalmente en la tabla VIll se han computado los porcentajes de todas o alguna respuesta equivocadas a las tres preguntas relativas a las ideas de los alumnos sobre el modelo cinético de los gases, al igual que se hizo con jas tres primeras preguntas.

\section{CONCLUSIONES}

Aun aceptando con cautela los resultados encontrados, dado que seria necesario ampliar la muestra de población escolar y al mismo tiempo, diseñar nuevos experimentos que intentaran verificar más rigurosamente la hipótesis emitida, se puede concluir que la mayor parie de los alumnos que inician los estudios de la Química en la enseñanza media tienen asumidas ideas divergentes sobre los gases, pues, mientras conservan rajces aristotélico-escolásticas en la concepción del gas como materia, sin embargo, tienen asimilados aspectos del modelo cinético actual de los gases, si bien aparecen también ideas estáticas soore las partículas gaseosas. Estas preconcepciones de los alumnos son de gran interés para el profesor de Quimica que desea desarrollar una metodologia activa de descubrimiento guiado coherente con la metodologia científica, pues, entendemos que la mayor parte de los esquemas conceptuales de nuestros alumnos en Química (ideas subre las reacciones químicas, concepto de molécula, etc...) son ignorados por el profesorado.

Este trabajo forma parte de una investigación más extensa cuyo objetivo esencial es of recer una aproximación a la ieoría atómicommolecular para alumnos que 
inician los estudios de Química en el Bachillerato, por el camino que históricamente ha sido más fácil, como es el conocimiento de los gases. Ello no significa restar importancia a la segunda lfnea de investigación que convergió con la química neumática a principios del siglo XIX: el estudio de las reacciones químicas entre sólidos y liquidos.

\section{REFERENCIAS BIBLIOGRAFICAS}

ARISTOTELES, 1973. Obras. Fisica. (Aguilar S.A., Madrid). AUSUBEL, D.P., 1978, Psicologia educativa. Un punto de vista cognitivo. (Trillas, México).

CARRASCOSA J. y GIL D., 1982. Los errores conceptuales en ia ensehanza de la Ftsics. I Un estudio de su persistencia en Primeros Jornadas de Investigación Didectica de $F$, y $Q$. Vajencia.

CARRASCOSA J., GIL D. y GONZALEZ A., 1982. Un nuevo tra tamiento de los errores conceptuaies en la ensefianza de la Fisica en Primeras Jornodas de Investigación Didóctica de $F$, y $Q$. Valencia.

DAVY T.. 1976, Boyle et sa loi, L'ocsualite chimique, p. 20.22.

GARRET, H.E., 1971. Estadística en Psicología y Educacion (Paj. dos, Buenos Aires).

GIL, D., 1981. Evolución del concepto de materia. (ICE de la Universidad de Valencia).

HELM, 1980. Stucents' conceptions of ideas in mechanics. Phys. Educ. Vol. 17, pp. 62-66.
HEWSON, P.W., 1981. A conceptual change approach to tearning science. Eur. J. Sci. Educ., Vol. 3, pp. 383-396.

HOLTON, G., 1973. Thematic origins of scientific thought. (Hatvard Univ. Pres. Cambridge, Mass.).

KUHN, T.S., 1971. Lo estructuro de las revoluciones cientificas. (Fondo de Cultura Economica. Mtxico).

NASH, L.K., 1970. Horvard Cose Histories in experimental Science. (Harvard Univ. Press., Cambridge, Mass.).

PIAGET, J., i97S. Introducción o la epistemologlo gentético. El pensamiento fisico. (Paidos, Buenos Aires).

PIAGET, J. Y INHELDER, B., 1971 . El desarrollo de los cantidades en el niflo. (Ed. Nova Terra, Barcelona).

TATON, R., 1958. Mistoire Generale des Sciences, Vol. II, p. 343. (Presses Universitaires de France, Paris).

VIENNOT, L., 1979. Spontaneous reasoning in elementary dynamics. Eur. J. Sci. Educ., 2, p. 205-221. 
TABLA J

1. El aire, el humo, etc... son ejemplos de gases. ¿Crres que pisan?

a) SI

b) NO

c) NO LO SE

\begin{tabular}{|c|c|c|c|c|c|c|}
\hline \multirow{2}{*}{ CURSO } & \multirow{2}{*}{$N^{\circ}$ RESP. } & \multicolumn{5}{|c|}{$\%$ RESPUESTAS } \\
\hline & & la) & (b) & Ic) & TOT. EQUI. & 5 \\
\hline $\begin{array}{l}5^{\circ} \\
6^{\prime \prime} \\
7^{\prime \prime} \\
8^{\prime \prime} \\
7^{\prime \prime} \\
2^{\prime \prime}\end{array}$ & $\begin{array}{l}36 \\
34 \\
65 \\
60 \\
73 \\
70\end{array}$ & $\begin{array}{l}30.6 \\
32.4 \\
60.0 \\
95.0 \\
90.4 \\
82.9\end{array}$ & $\begin{array}{r}63.9 \\
67.6 \\
40.0 \\
3.3 \\
8.2 \\
14.3\end{array}$ & $\begin{array}{l}5.5 \\
0 \\
0 \\
1.7 \\
1.4 \\
2.8\end{array}$ & $\begin{array}{r}69.5 \\
67.6 \\
40.0 \\
5.0 \\
9.6 \\
17.1\end{array}$ & $\begin{array}{l}7.7 \\
8.0 \\
6.1 \\
2.8 \\
3.4 \\
4.5\end{array}$ \\
\hline
\end{tabular}

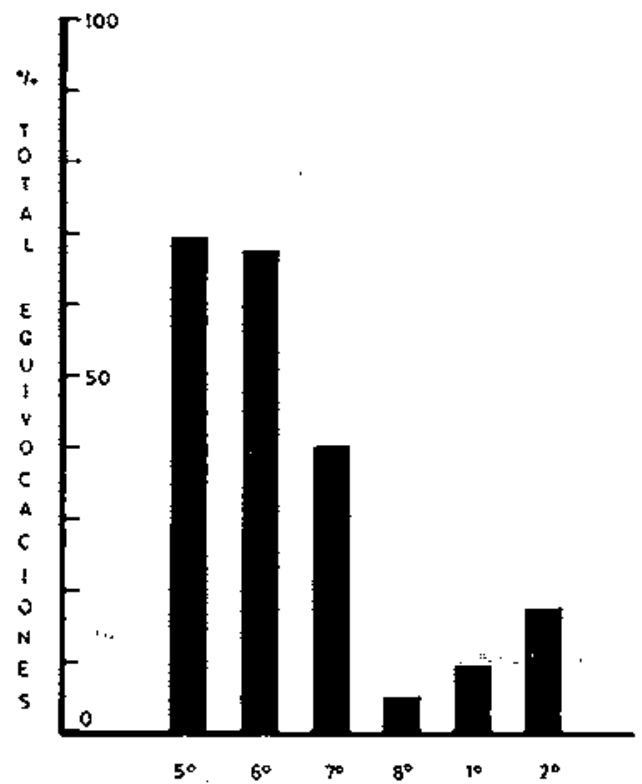

Fig. 1

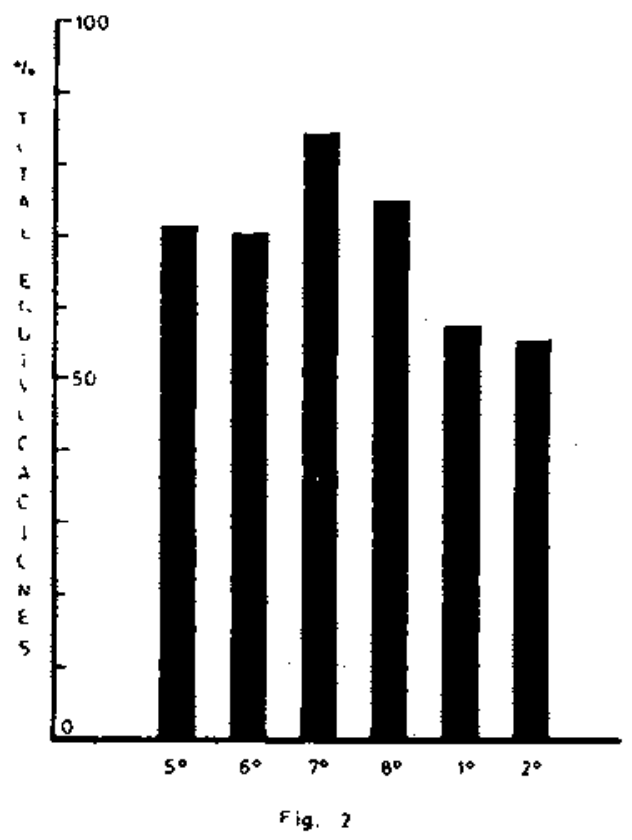

IABLA II

2. Supongamos que calentamos agua en una «ollan cerrada (doncie no puede tstaparse nada) hasta que no quede tiquido. ¿Cuando srees que pesara la olla?
a) Cuando contiene tiquido
b) Cuando no quede liquido
c) Pesarán igual
d) No lo sé

\begin{tabular}{|c|c|c|c|c|c|c|c|}
\hline \multirow{2}{*}{ CURSO } & \multirow{2}{*}{$N^{\circ}$ RESP. } & \multicolumn{6}{|c|}{$\%$ RESPUESTAS } \\
\hline & & $2 a$ & $2 b$ & 20 & $2 d$ & TOT EQUI. & $\mathbf{s}$ \\
\hline $\begin{array}{l}5^{\circ} \\
6^{\circ} \\
7^{\circ} \\
8^{\circ} \\
1^{\circ} \\
2^{\circ}\end{array}$ & $\begin{array}{l}36 \\
34 \\
65 \\
60 \\
73 \\
70\end{array}$ & $\begin{array}{l}54.3 \\
58.8 \\
78.5 \\
65.0 \\
45.2 \\
40.0\end{array}$ & $\begin{array}{r}11.4 \\
11.8 \\
6.1 \\
8.3 \\
3.5 \\
2.9\end{array}$ & $\begin{array}{l}28.6 \\
29.4 \\
15.4 \\
25.0 \\
42.5 \\
44.3\end{array}$ & $\begin{array}{l}5.7 \\
0 \\
0 \\
1.7 \\
6.8 \\
12.8\end{array}$ & $\begin{array}{l}71.4 \\
70.6 \\
84.6 \\
75.0 \\
57.5 \\
55.7\end{array}$ & $\begin{array}{l}7.6 \\
7.8 \\
4.4 \\
5.6 \\
5.8 \\
5.9\end{array}$ \\
\hline
\end{tabular}




\section{TABLA III}

3. Supongamos que calentamos en un cazo tapado un poco de agua hasta que se convierte toda en vapor (gas). ¿Cuál crees que será el volumen ocupado por el vapor?

a) el mismo que ci det biquido

volumen det liquido

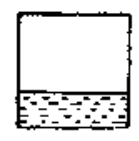

b) el mismo pero ocupado la parte alta

c) todo el recipiente

d) no to sé
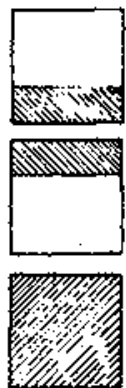

\begin{tabular}{|c|c|c|c|c|c|c|c|}
\hline \multirow{2}{*}{ CURSO } & \multirow{2}{*}{$N^{*}$ RESP. } & \multicolumn{6}{|c|}{ \% RESPUESTAS } \\
\hline & & $3 a$ & $3 b$ & $3 c$ & $3 d$ & TOT EQUI. & s \\
\hline 50 & 36 & 8.3 & 36.1 & 38.9 & 16.7 & 61.1 & 8.1 \\
\hline $6^{\circ}$ & 34 & 2.9 & 58.8 & 35.3 & 2.9 & 64.7 & B.2 \\
\hline$\%$ & 65 & 1.5 & 44.0 & 52.3 & 1.5 & 47.7 & 6.2 \\
\hline $8^{\prime \prime}$ & 60 & 1.7 & 36.7 & 58.3 & 3.3 & 41.7 & 6.4 \\
\hline to & 73 & 0 & 32.9 & 67.1 & 0 & 33.9 & 5.6 \\
\hline $2^{0}$ & 70 & 0 & 32.9 & 62.9 & 4.2 & 37.1 & 5.8 \\
\hline
\end{tabular}

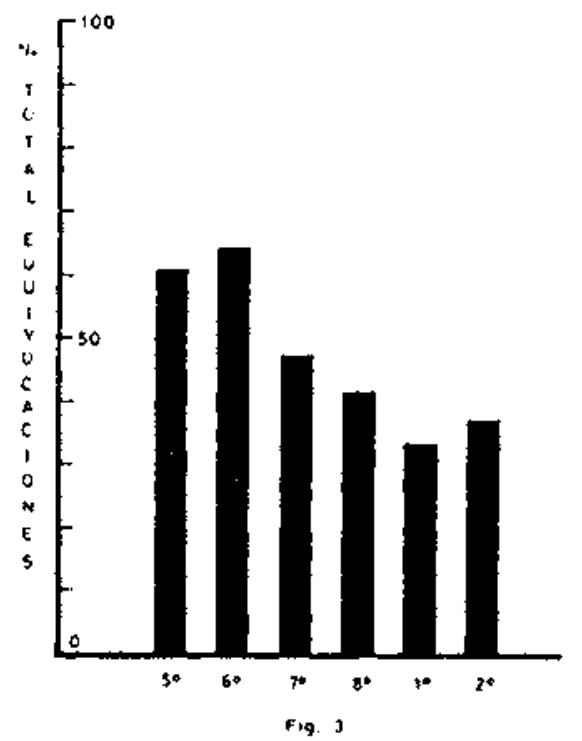

TABLA IV

\begin{tabular}{|c|c|c|c|}
\hline CURSO & $N^{\circ}$ RESP. & \% RESP. EQ. & $s$ \\
\hline $5^{\circ}$ & 36 & 100 & - \\
$6^{\circ}$ & 34 & 100 & - \\
$7^{\circ}$ & 65 & 95.4 & 2.6 \\
$8^{\circ}$ & 60 & 81.7 & 5.1 \\
$1^{\circ}$ & 73 & 69.9 & 5.4 \\
$2^{\circ}$ & 70 & 72.9 & 5.3 \\
\hline
\end{tabular}




\section{TABLA V}

4. Consideras que los gases están formados por trozos continum (csmw p.c. un ovillo de lana) o piensas que los gases lo forman muchos trozos pequetios (como p.e. la arena).
a) AJgo continuo
b) Trozos pequerios
c) No to sé.

\begin{tabular}{|c|c|c|c|c|c|c|c|}
\hline \multirow{2}{*}{ CURSO } & \multirow{2}{*}{$N^{*}$ RESP. } & \multicolumn{6}{|c|}{$\%$ RESPUESTAS } \\
\hline & & $4 a$ & $4 b$ & $4 c$ & $4 d$ & TOT EQUI. & $s$ \\
\hline $\begin{array}{l}5^{\circ} \\
6^{\circ} \\
7^{\circ} \\
8^{\circ} \\
1^{\circ} \\
2^{\circ}\end{array}$ & $\begin{array}{l}36 \\
34 \\
65 \\
60 \\
73 \\
70\end{array}$ & $\begin{array}{l}36.1 \\
17.6 \\
33.8 \\
20.0 \\
34.2 \\
22.9\end{array}$ & $\begin{array}{r}44.4 \\
73.6 \\
58.5 \\
75.0 \\
61.6 \\
68.6\end{array}$ & $\begin{array}{r}19.4 \\
8.8 \\
7.7 \\
5.0 \\
4.1 \\
8.6\end{array}$ & $\begin{array}{l}55.6 \\
26.4 \\
41.5 \\
25.0 \\
38.4 \\
31.4\end{array}$ & $\begin{array}{l}8.3 \\
7.6 \\
6.1 \\
5.6 \\
5.7 \\
5.5\end{array}$ & \\
\hline
\end{tabular}
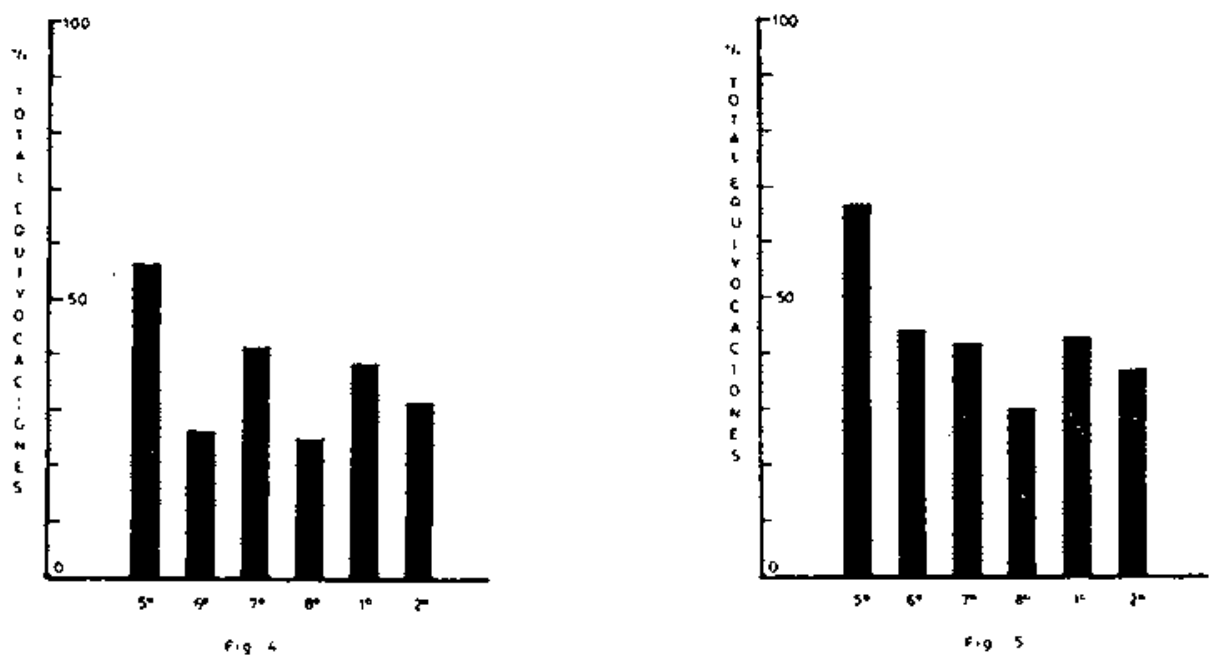

TABI.A V1

5. Una de las propiedades más conocidas de los gases es su compresión fque consiste ẹn poder reducir el volumen que ocupa al presionar sobre él, como se puede comprobar con una jeringuilla\}.

¿Cuál de estas situaciones crees que se produce?

a) Las particulas existentes reducen so samaño

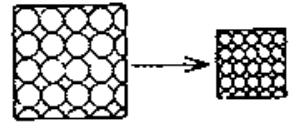

b) Los gases son como esponjas (todo continuo) que al apretar se comprimen

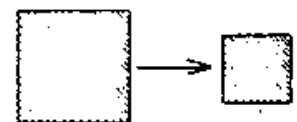

c) Las particulas que to iorman dejan espacios libres «huecos" que al apretar se reducen

d) No lo sé

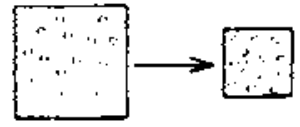

\begin{tabular}{|c|c|c|c|c|c|c|c|}
\hline \multirow{2}{*}{ CURSO } & \multirow{2}{*}{$N^{\circ}$ RESP. } & \multicolumn{6}{|c|}{$\%$ RESPUESTAS } \\
\hline & & Sa & $5 b$ & $\mathrm{sc}$ & Sd & TOT EQUI. & $s$ \\
\hline $\begin{array}{l}5^{\circ} \\
6^{\circ} \\
7^{\circ} \\
8^{\circ} \\
1^{\circ} \\
2^{\circ}\end{array}$ & $\begin{array}{l}36 \\
34 \\
65 \\
60 \\
73 \\
70\end{array}$ & $\begin{array}{r}27.8 \\
17.6 \\
21.5 \\
13.3 \\
13.7 \\
5.7\end{array}$ & $\begin{array}{r}27.8 \\
26.5 \\
18.5 \\
8.3 \\
20.6 \\
24.3\end{array}$ & $\begin{array}{l}33.3 \\
55.9 \\
58.5 \\
70.0 \\
57.5 \\
62.9\end{array}$ & $\begin{array}{l}11.1 \\
0 \\
1.5 \\
8.3 \\
8.2 \\
7.2\end{array}$ & $\begin{array}{l}66.7 \\
44.1 \\
41.5 \\
30.0 \\
42.5 \\
37.1\end{array}$ & $\begin{array}{l}7.9 \\
8.5 \\
6.1 \\
5.9 \\
5.8 \\
5.8\end{array}$ \\
\hline
\end{tabular}




\section{TABLA VII}

6* Tú sabes que los gases se mueven, putes en una habiación al destapar un perfume, se kiucle en loda ella. Ahora, ¿cómo crees que se mueven los gases?

a) como si fuera un muelle

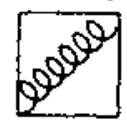

b) todas las particulas se mueven conjuntamente pasando cada vez por un sitio dislinıo de la habitación:

c) cada pariicula se mueve constanlemente hacia cualquier direccion:

d) no lo sé

\begin{tabular}{|c|c|c|c|c|c|c|c|}
\hline \multirow{2}{*}{ CURSO } & \multirow{2}{*}{$N^{\prime \prime}$ RESP. } & \multicolumn{6}{|c|}{ \% RESPUESTAS } \\
\hline & & $6 a$ & $6 b$ & $6 c$ & $6 d$ & TOT.EQU1. & s \\
\hline 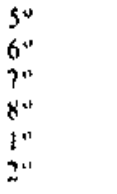 & $\begin{array}{l}36 \\
34 \\
65 \\
60 \\
73 \\
70\end{array}$ & $\begin{array}{l}0 \\
2.9 \\
4.6 \\
0 \\
0 \\
2.9\end{array}$ & $\begin{array}{l}33.3 \\
14.7 \\
13.8 \\
30.0 \\
15.1 \\
30.0\end{array}$ & $\begin{array}{l}63.9 \\
79.4 \\
78.5 \\
63.3 \\
82.2 \\
72.9\end{array}$ & $\begin{array}{l}2.8 \\
0 \\
3.1 \\
6.7 \\
2.7 \\
4.2\end{array}$ & $\begin{array}{l}36.1 \\
20.6 \\
21.6 \\
36.7 \\
18.8 \\
27.1\end{array}$ & $\begin{array}{l}8.0 \\
6.9 \\
5.1 \\
6.2 \\
4.6 \\
5.3\end{array}$ \\
\hline
\end{tabular}

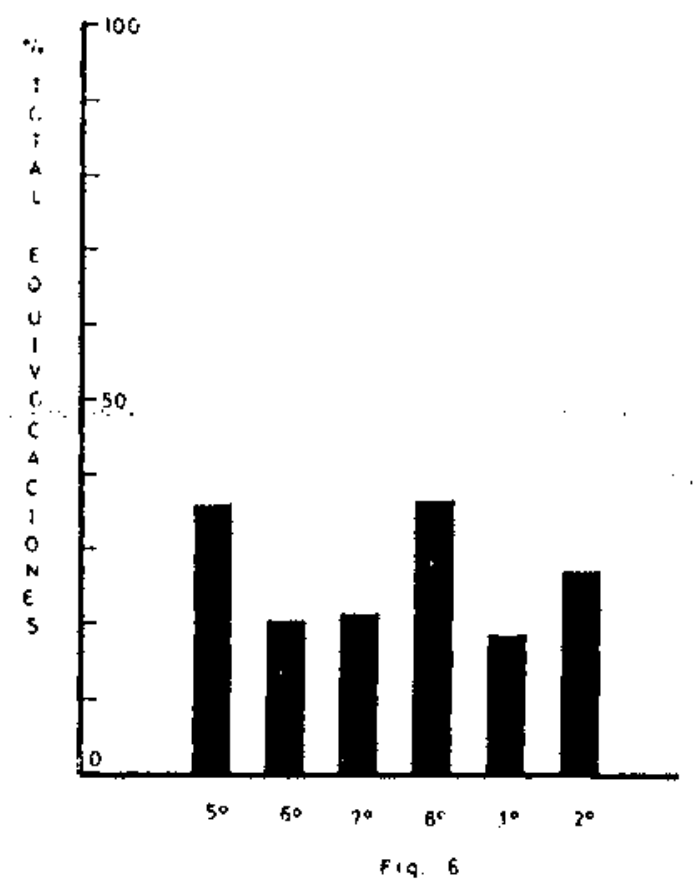

TABLA VIII

\begin{tabular}{|c|c|c|c|}
\hline CURSO & $N^{\circ}$ RESP. & $\begin{array}{c}\text { \% RESP. EQ. } \\
\text { (TOD. O ALG.) } \\
\text { (cuest. } 4^{\circ} .5^{\circ} \text { y 6*) }\end{array}$ & $\mathrm{s}$ \\
\hline $5^{\circ}$ & 36 & 80.6 & 6.6 \\
$6^{\circ}$ & 34 & 67.6 & 8.0 \\
$7^{\circ}$ & 65 & 73.8 & 5.4 \\
$8^{\circ}$ & 60 & 63.3 & 6.2 \\
$1^{\circ}$ & 73 & 71.2 & 5.3 \\
$2^{\circ}$ & 70 & 61.4 & 5.8 \\
\hline
\end{tabular}

